\title{
Investigations on Various Studies of Triglycine Sulphophosphate Crystals Doped With Cesium Chloride
}

\author{
S.Gracelin Juliana ${ }^{1}$, P.Selvarajan ${ }^{2}$ and S.Perumal ${ }^{3}$ \\ ${ }^{l}$ Department of Physics, Nazareth Margoschis College, Nazareth-628617, Tamilnadu, India. \\ ${ }^{2}$ Department of Physics, Aditanar College of Arts and Science, Tiruchendur-628216, Tamilnadu, India. \\ ${ }^{3}$ Physics Research Centre, S.T. Hindu College, Nagercoil-629003, India.
}

\begin{abstract}
Single crystals of triglycine sulpho-phosphate (TGSP) doped with cesium chloride were grown by slow evaporation technique. From single crystal XRD studies, it is observed that the grown crystals crystallize in monoclinic structure. Mechanical studies were performed by measuring microhardness of the samples at different loads. Second order NLO studies were carried out to understand the second order NLO activity. The ferroelectric nature of the sample has been analyzed by measuring dielectric constant and loss factor. Third order NLO parameters have been measured by Z-scan technique. LDT values of the samples have been measured using a Nd:YAG laser and the results are discussed.
\end{abstract}

Keywords: TGSP crystal, doping, solution growth, single crystal, characterization, LDT, SHG

\section{Introduction}

It is well known that when glycine combines with sulphuric acid in the molar ratio 3:1, Triglycine Sulphate (TGS) crystal is formed and it is a suitable material for developing detectors of infrared radiation, astronomical telescopes, military systems and target faces in vidicons based on the pyroelectric effect. Like TGS crystal, Triglycine Sulpho-Phosphate(TGSP) crystal is also a ferroelectric and pyroelectric material which could be a suitable material for room temperature infrared detection and the other applications. Growth and studies of TGSP crystals was reported by Ravikumar et al. [1]. It is obtained by partial substitution of sulphate ions with phosphate ions in TGS crystal and this partial substitution phosphate ions delays microbial contamination during the growth and avoids depolarization with time[2,3]. Aparna Saxena et al. reported the various characterization studies on phosphoric acid doped TGS (TGSP) crystals and observed that the dielectric constant of TGSP crystal is low as that of TGS crystal and hence increases the pyroelectric figure of merit $[4,5]$. Doping crystals with various kinds of dopants influences the solubility, growth rate, morphology, structural, electrical and other properties of the crystals [6-10]. In this work, an inorganic material like cesium chloride is added as a dopant into TGSP crystal to modify its physical and chemical properties. Hence, the aim of this work is to grow the crystals of undoped and cesium chloride doped TGSP and to characterize the grown crystals.

\section{Preparation of samples}

Saturated aqueous solution of TGSP was prepared using Analar Reagent (AR) grade of glycine, concentrated sulphuric acid and concentrated ortho-phosphoric acid in the molar ratio of 3:0.8:0.2. Synthesized Triglycine Sulpho-Phosphate(TGSP) salt was obtained by heating the saturated solution of TGSP at $50{ }^{\circ} \mathrm{C}$. To obtain the doped TGSP salt, 1 mole $\%$ of cesium chloride was added to aqueous solution of TGSP. The synthesized salt of TGSP and the doped salt were re-crystallized twice for further purification. Growth of undoped and cesium chloride doped TGSP crystals was carried out by solution method with slow evaporation technique. Using the synthesized salts and double distilled water as the solvent, saturated solutions were prepared solutions and stirred well using a magnetic stirrer, filtered using the filter papers. Then, the filtered solutions were kept in the growth vessels separately covered with the porous papers for the growth of the crystals.

\subsection{Crystal structure of the grown crystals}

\section{Results and discussion}

Single crystal XRD studies were carried out to find the crystal structure of the samples and these studies were done using an ENRAF NONIUS CAD4 diffractometer with MoK radiation $(\lambda=0.71073 \AA$. The obtained XRD data for the cesium chloride doped TGSP crystal are $a=9.237(4) \AA \quad, b=12.695(3) \AA$ 5.863(2) $\AA$, $\alpha=90^{\circ}, \beta=111.64^{\circ}(2), \gamma=90^{\circ}$ and $V=639.11(3) \AA^{3}$. From the obtained data, it is observed that the cesium chloride doped TGSP crystal crystallizes in monoclinic structure with the space group $\mathrm{P} 2{ }_{1}$. The 
values of lattice parameters obtained in this work are observed to be in close agreement with the crystallographic data of the undoped TGSP crystal [11]. The results indicate that the crystal structure of both undoped and cesium chloride doped TGSP crystals is the same.

\subsection{Kurtz powder method}

Kurtz powder method was used to find the second harmonic generation efficiency of the samples [12]. The powdered sample was illuminated using a Nd: YAG laser with the first harmonics output of $1064 \mathrm{~nm}$ with pulse width of $8 \mathrm{~ns}$ and repetition rate $10 \mathrm{~Hz}$. The second harmonics signal, generated in the crystal was confirmed from the emission of green radiation by the crystal. The SHG radiation of $532 \mathrm{~nm}$ green light was collected by a photomultiplier tube after being monochromated to collect only $532 \mathrm{~nm}$ radiation. The optical signal incident on the PMT was converted into voltage output at the CRO. The obtained values of relative SHG efficiency are 1.33 and 1.16 respectively for undoped and cesium chloride doped TGSP crystals.

\subsection{Hardness measurement}

Hardness of the grown crystals has been tested by microhardness analysis. This test was carried out using a Shimadzu Vickers microhardness tester fitted with a diamond indenter attached to an incident light microscope. Microhardness number was determined using the relation $\mathrm{H}_{\mathrm{v}}=1.8544 \mathrm{P} / \mathrm{d}^{2}$ where $\mathrm{P}$ is the applied load and $\mathrm{d}$ is the average diagonal indentation[13-15]. The variation of hardness number with the applied load for the samples is shown in the figure 1. It is observed that the hardness number increases with increase of applied load for both the samples. The increasing part of the curve is due to the reverse indentation size effect and when the hardness decreases, it is due to the direct indentation size effect. When an inorganic material like cesium chloride is added as the dopant, it seems that the hardness of TGSP crystal increases.

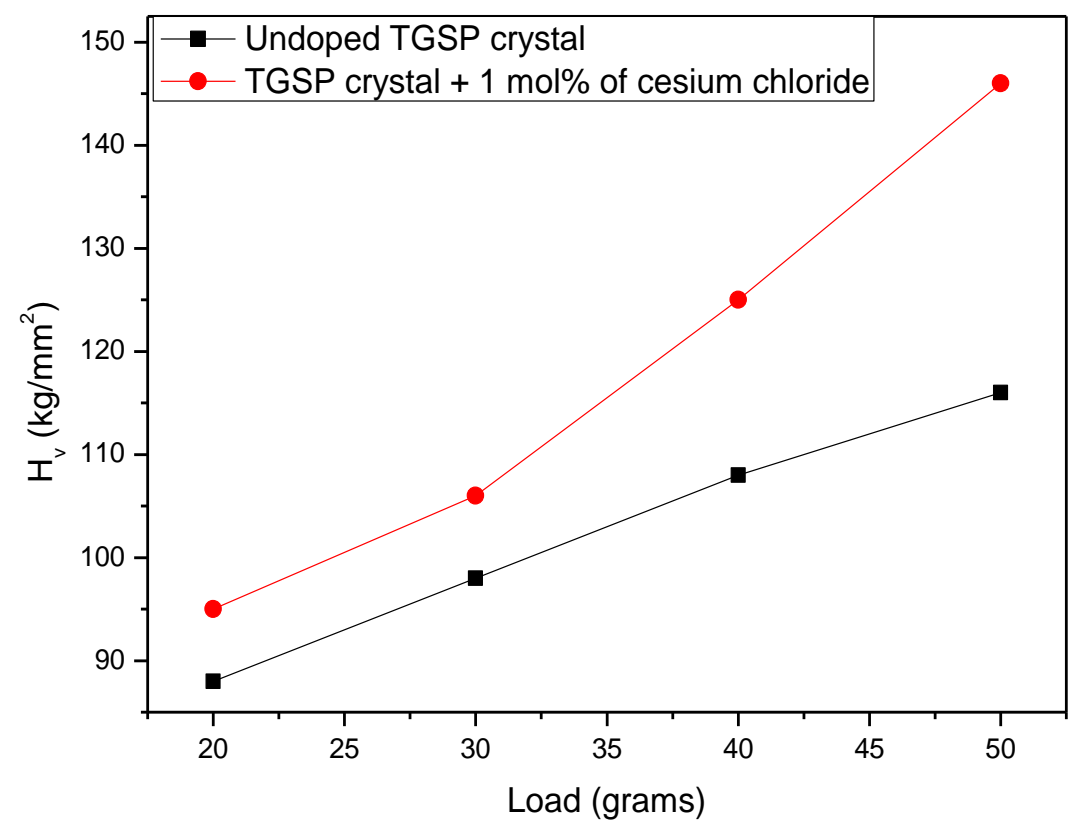

Fig.1: Variation of hardness number with the applied load for undoped and cesium chloride doped TGSP crystals

\subsection{Measurement of dielectric constant and loss factor}

The dielectric constant is one of the basic electrical properties of solids. The measurement of dielectric constant and loss as a function of frequency and temperature is of interest both from theoretical point of view and from the applied aspects. The dielectric constant and loss factor of the samples were measured using the parallel plate capacitor method at various temperatures ranging from 30 to $70{ }^{\circ} \mathrm{C}$ using an Agilent $4284 \mathrm{~A}$ LCR meter at different frequencies ranging from $10^{2}$ to $10^{6} \mathrm{~Hz}$. Opposite faces of the sample crystals were coated with good quality silver paste to obtain a good conductive surface layer. The variations of dielectric constant and dielectric loss factor as a function of temperature at the constant frequency of $10^{3} \mathrm{~Hz}$ are presented in the figures 2 and 3. The dielectric mechanism of a solid consists of contributions from electronic, ionic, dipolar and 
space charge polarizations, each dominating in a particular frequency range. It is established that the space charge polarization is very predominant at lower frequencies. Low value of dielectric loss indicates that the grown crystals are good quality dielectric materials[16,17]. When TGSP crystals are doped with cesium chloride, the dielectric constant and loss factor seem to be decreasing with temperature. The dielectric constant and loss of the samples at the transition temperature $\left(\mathrm{T}_{\mathrm{c}}\right)$ show finite values and the observed value of Curie temperature for these samples is $51{ }^{\circ} \mathrm{C}$. The values of dielectric constant and loss are found to be less at Curie temperature for the cesium chloride doped TGSP crystal compared with the values of pure TGSP crystal. This may be due to presence of metal dopants in the TGSP crystals. It is observed from the results that the Curie temperature is not changed when cesium chloride is added as the dopant into TGSP crystal.

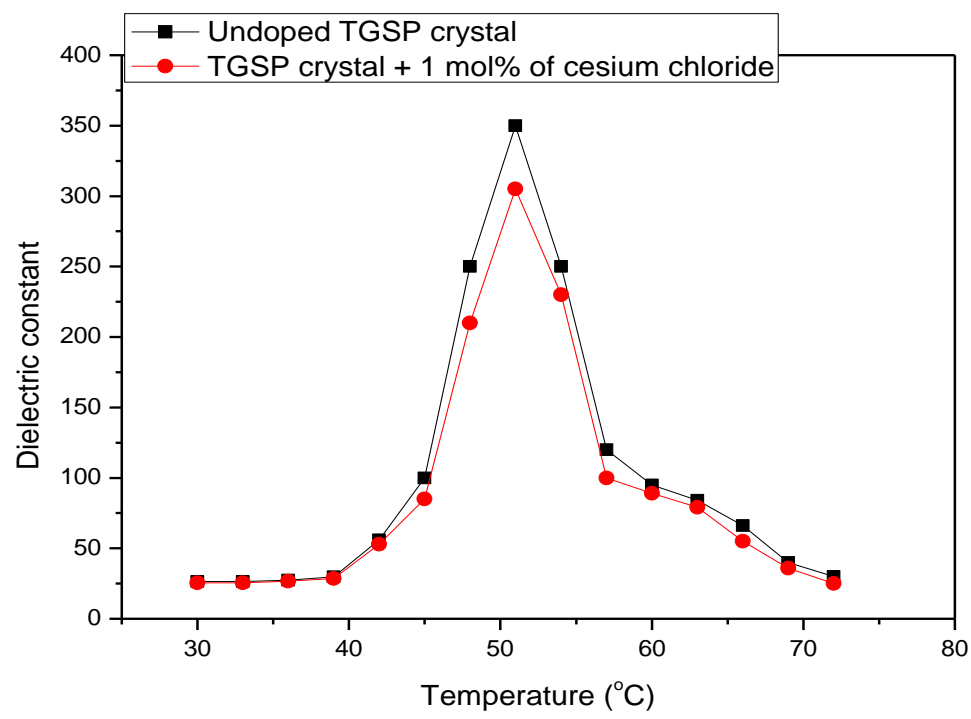

Fig.2: Temperature dependence of dielectric constant for undoped and cesium chloride doped TGSP crystals at a frequency of $1000 \mathrm{~Hz}$

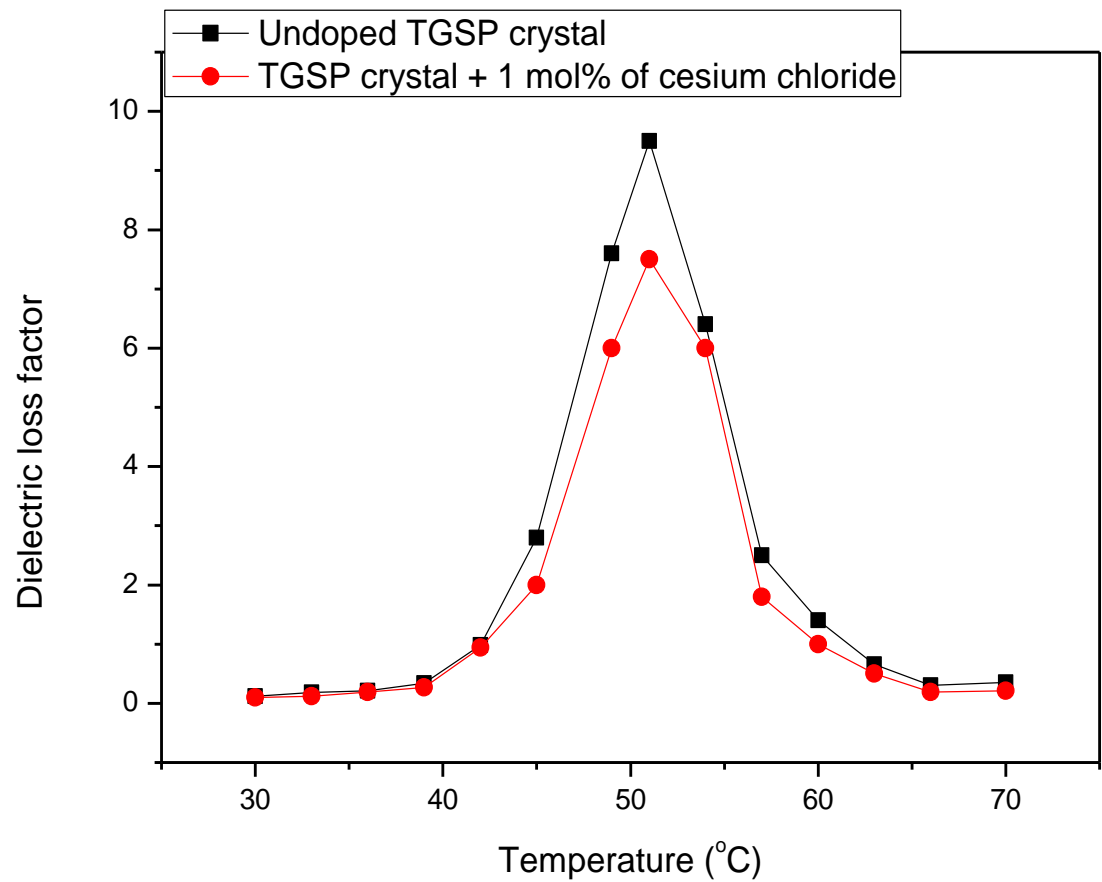

Fig.3: Temperature dependence of dielectric loss factor for undoped and cesium chloride doped TGSP crystals at a frequency of $1000 \mathrm{~Hz}$ 


\subsection{LDT measurement}

Laser damage threshold (LDT) is an important factor which affects the applications of optical applications. For nonlinear optical applications, one of the most important considerations and criteria in the choice of material is its tolerance and resistance to laser damage to perform as a device for NLO applications. Since high optical intensities are involved in nonlinear processes, NLO materials must be able to withstand high power intensity. Laser Damage Threshold values of grown crystals were measured using the Q-switched NdYAG laser and duration of the pulse is $10 \mathrm{~ns}$. The position of a convex lens is placed between the laser and the crystal. The focal length of a convex lens is $300 \mathrm{~mm}$, and the distance between the lens and the laser is $150 \mathrm{~mm}$. The diameter of the laser beam is $1 \mathrm{~mm}$. The laser damage threshold value depends on a number of laser parameters such as longitudinal and transverse mode structure. In this experiment, lens and crystal positions are fixed and the laser pulse energy was increased until a visible spot was seen at the crystal surface. The crystal is mounted on a sample holder and slightly kept away from the focal spot of the beam to avoid any possible damage. Laser operation can be controlled by Gaussian remote control back panel where the energy/volts button controls the output energy of the laser by varying the charge voltage in the flash lamp. The damage occurring on the surface of the sample is observed with the help of scattered radiation of laser from its surface. The laser beam is made collinear with the Nd: YAG laser beam such that it passes through the same spot where the damage is being affected. A sharp reduction in the intensity of the transmitted laser beam gives the laser damage threshold value. LDT value is the maximum permissible power that can withstand in a particular crystal and it is determined using the formula $\mathrm{P}=\mathrm{E} / \tau \pi \mathrm{r}^{2}$ where $\mathrm{E}$ is the energy in $\mathrm{mJ}, \tau$ is the pulse width, $\mathrm{r}$ is radius of the spot in mm. The obtained values of LDT for the undoped and cesium chloride doped TGSP crystals are 0.86 $\mathrm{GW} / \mathrm{cm}^{2}$ and $0.98 \mathrm{GW} / \mathrm{cm}^{2}$ respectively.

\subsection{Measurement of third-order NLO parameters}

Third-order nonlinear optical (NLO) parameters are nonlinear refractive index $\left(\mathrm{n}_{2}\right)$, nonlinear susceptibility $\left(\chi^{(3)}\right)$ and nonlinear absorption coefficient $(\beta)$ of the samples and these values can be found by $Z$ scan technique. The sign of the nonliner refractive index can also be found by Z-scan technique. The study of nonlinear refraction by the Z-scan method depends on the position $(Z)$ of the thin sample under the investigation along a focused Gaussian laser beam and moving sample along the propagation of direction $\mathrm{Z}$ in to the focal region of focused laser beam and conversion of phase distortion into amplitude distortion during the propagation[18,19]. .Measurements of the normalized transmittance by varying the sample position (Z) have been made for the grown crystals in open and closed aperture modes. In the closed aperture method, an aperture is placed to prevent some of the light from reaching the detector. A lens focuses a laser to a certain point, and after this point the beam naturally defocuses. After a further distance an aperture is placed with a detector behind it. The aperture causes only the central region of the cone of light to reach the detector. The detector is sensitive to any focusing or defocusing that a sample may induce. The sample is typically placed at the focus point of the lens, and then moved along the Z-axis. Open aperture method is similar to the above method, however the aperture is removed or enlarged to allow all the light to reach the detector. This is used in order to measure the nonlinear absorption coefficient. The laser light intensities, transmitted across the sample, are measured as a function of sample position in the Z-direction with respect to the focal plane either through a closed aperture $(\mathrm{CA})$ or open aperture $(\mathrm{OA})$ in order to resolve the nonlinear refraction and absorption coefficients.

The Z-scan curves are characterized by a prefocal transmittance maximum (peak) followed by a postfocal transmittance minimum (valley) intensity. The transmission difference between peak and valley $\left(\Delta \mathrm{T}_{\mathrm{p}-\mathrm{v}}\right.$ ) is written in terms of phase shift.

$$
\Delta T_{p-v}=0.406(1-s)^{0.25}|\Delta \phi|
$$

Linear transmittance aperture $(\mathrm{S})$ is calculated using the relation

$$
\mathbf{S}=1-\exp \left(\frac{-2 r_{\alpha}^{2}}{\omega_{\alpha}^{2}}\right)
$$

where $r_{a}$ is the radius of the aperture and $\omega_{a}$ is the beam radius at the aperture. The third-order nonlinear refractive index $\left(\mathrm{n}_{2}\right)$ of the crystal was calculated by following the relation.

$$
\mathrm{n}_{2}=\Delta \phi /\left(\mathrm{K} \mathrm{I}_{\mathrm{o}} \mathrm{L}_{\text {eff }}\right)
$$

where $I_{0}$ is the intensity of the laser beam at the focus $(Z=0)$ and $K=2 \pi / \lambda \quad(\lambda$ is the wavelength of laser beam). The effective thickness can be calculated using the relation

$$
L_{\text {eff }}=[1-\exp (-\alpha L)] / \alpha
$$

where $\alpha$ is the linear absorption coefficient and $L$ is the thickness of the

sample. The nonlinear absorption coefficient $(\beta)$ can be calculated using the following relation

National Conference on Current Advancements in Physics $3^{\text {rd }} \& 4^{\text {th }}$ February 2017 


$$
\boldsymbol{\beta}=\frac{2 \sqrt{2} \Delta \mathrm{T}}{\mathrm{I}_{0} \mathrm{~L}_{\mathrm{eff}}}
$$

where $\Delta \mathrm{T}$ is the one peak value at the open aperture $\mathrm{Z}$-scan curve. The value of $\beta$ will be negative for saturable absorption and positive for two photon absortion process. The real and imaginary parts of the third order nonlinear optical suceptibility $\left(\chi^{(3)}\right)$ are defined as

$$
\begin{aligned}
& \text { Real part of } \chi^{(3)}=\left(10^{-4} \varepsilon_{0} c^{2} n_{0}^{2} n_{2}\right) / \pi \quad \text { (esu) } \\
& \text { Imaginary part of } \chi^{(3)}=\left(10^{-2} \varepsilon_{0} c^{2} n_{0}^{2} \lambda \beta\right) / 4 \pi^{2} \quad \text { (esu) }
\end{aligned}
$$

Absolute value of $\chi^{(3)}=\left[\left\{\text { Real part of } \chi^{(3)}\right\}^{2}+\left\{\text { Imaginary part of } \chi^{(3)}\right\}^{2}\right]^{1 / 2}$ (esu). Here $\varepsilon_{0}$ is the vacuum permitivity, $\mathrm{n}_{\mathrm{o}}$ is the linear refractive index of the sample and $\mathrm{c}$ is the velocity of the light in vacuum. Using the above equations, nonlinear refractive index, third-order susceptibility and the nonlinear absorption coefficient of the crystals could be determined.

In Z-scan method, He-Ne laser $(\lambda=632.8 \mathrm{~nm})$ was used as the light source and focused by a lens of $22.5 \mathrm{~cm}$ focal length. The open aperture and closed aperture curves for the cesium chloride doped TGSP crystal are given in the figures 4 and 5. The obtained values of third order NLO parameters are provided in the table 1. It is observed from results that the sample has negative nonlinear refractive index because the sample shows transmittance peak followed by transmittance valley and therefore the sample shows the self-defocusing behaviour. The results of nonlinear absorption and nonlinear refraction of the sample reveal that these phenomena always coexist as they result from the same physical mechanisms.

Table 1: Values of third-order NLO parameters of cesium chloride doped TGSP crystal

\begin{tabular}{|c|c|c|}
\hline $\begin{array}{c}\text { Nonlinear refractive } \\
\text { index }\left(\mathrm{n}_{2}\right) \\
\times 10^{-10} \\
\left(\mathrm{~cm}^{2} / \mathrm{W}\right)\end{array}$ & $\begin{array}{c}\text { Nonlinear absorption } \\
\text { coefficient }(\beta \square) \\
\square \times 10^{5} \\
(\mathrm{~cm} / \mathrm{W})\end{array}$ & $\begin{array}{c}\text { Third-order nonlinear } \\
\text { susceptibility }\left(\chi^{(3)}\right) \\
\times 10^{-6} \\
(\mathrm{esu})\end{array}$ \\
\hline 2.74 & 6.83 & 4.72 \\
\hline
\end{tabular}

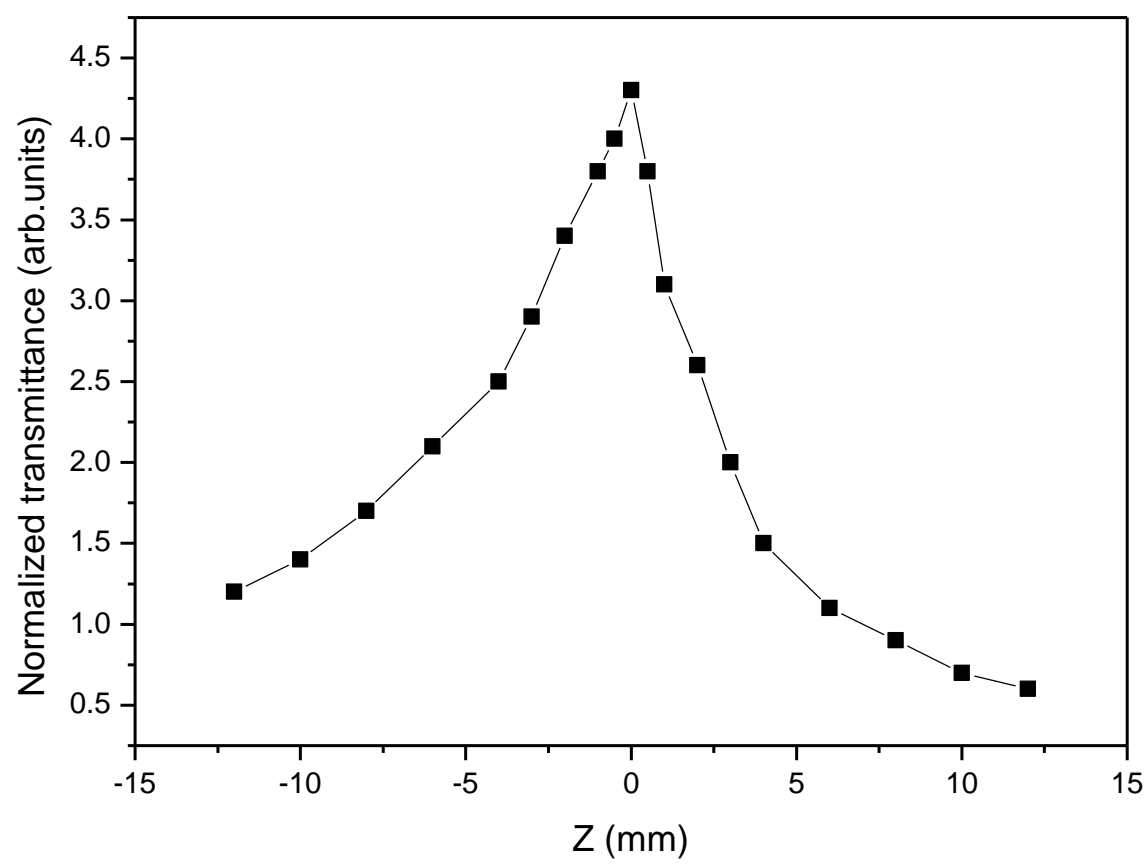

Fig.4: Open aperture Z-scan curve for cesium chloride doped TGSP crystal 


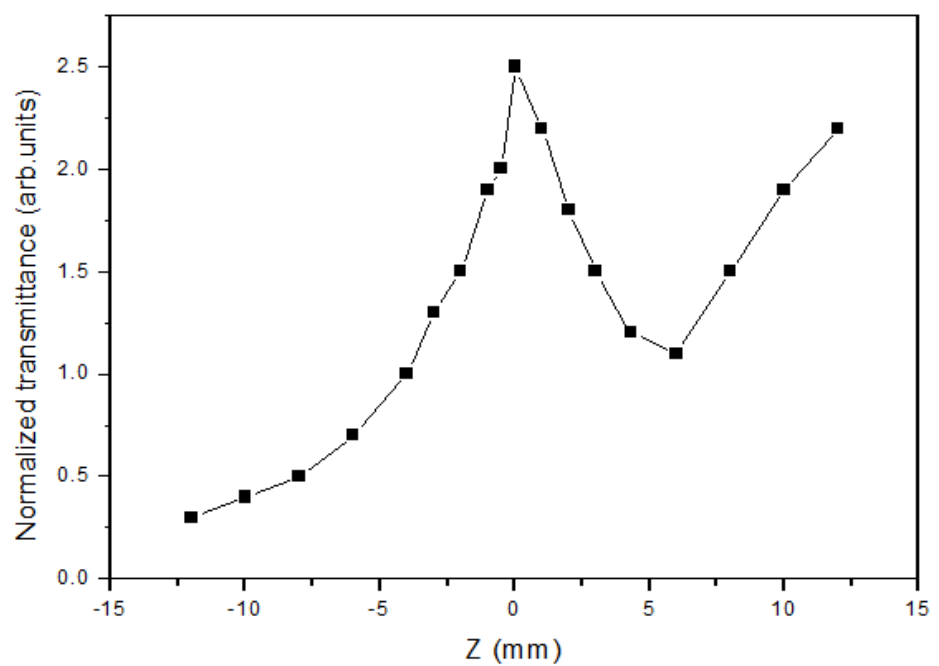

Fig.5: Closed aperture Z-scan curve for cesium chloride doped TGSP crystal

\section{Conclusions}

Undoped and cesium chloride doped triglycine sulphophosphate crystals were grown by slow evaporation technique. XRD studies indicates the monoclinic structure of the crystals. The hardness of the samples has been measured by Vickers hardness test. Both the samples are observed to be ferroelectric and the transition temperature is found to be at $51{ }^{\circ} \mathrm{C}$. Hardness and LDT values of the samples were found out and these values seem to be increased when TGSP samples are doped with cesium chloride. SHG efficiency of the samples have been measured by Kurtz powder method. Z-scan method was used to determine the third order NLO parameters.

\section{Acknowledgements}

The authors are thankful to SAIF, IIT Chennai, VIT, Vellore, Crescent Engineering College, Chennai, St.Joseph's College, Trichy for the scientific supports.The authors are grateful to the managements of Aditanar College of Arts and Science,Tiruchendur, Nazareth Margoschis College, Nazareth and S.T. Hindu College, Nagercoil for the encouragement given to us to carry out the research work.

[1]. G.Ravikumar, S.Anbukumar, P.Ramasamy, J.Cryst.Growth, 133(1993)212.

[2]. Drozhdin, O.M. Golitsyna, A.I. Nikishina, A. I, A.M. Kostsov, Ferroelectrics, 373(2008)93.

[3]. G.Arunmozhi, R.Jayavel, C.Subramanin, Mater. Chem.and Phys. 50(1997) 57.

[4]. Aparna Saxena, Vinay Gupta, K.Sreenivas, J.Cryst. Growth, 263( 2004) 192.

[5]. Aparna Saxena, M.Fahim, Vinay Gupta and K. Sreenivas, J.Phy.D.Appl.Phys., 36(2003)3168.

[6]. N.Drozhdin, O.M.Golitsyna and A.I.Nikishina, Bull. Russian Acad.Sci.Phys, 71 (2007) 1419.

[7]. Alexander McPherson, Alexander J . Malkin, Yu. G. Kuznetsov, Stanley Koszelak, J. Cryst.Growth 168(1996) 74.

[8]. B.Milton and S.Jerome Das, J.Cryst. Growth, 279(2005)383.

[9]. S.Ramlingom, J.Podder, S.Narayana Kalkura, G.Bocelli, J.Crystal Growth, 247(2003)523.

[10]. P.Selvarajan, A.Sivadhas, T.H.Freeda, C.K.Mahadevan, Physica B, 403 (2008) 4205-4208.

[11]. G.Ravi, S.Anbukumar, C. Subramanian, P.K.C.Pillai and P.Ramasamy, Ferroelectrics 166(1995)47.

[12]. D. Shanthi, P. Selvarajan, R. Jothi Mani, Optik 125 (2014) 2531-2537.

[13]. P.Selvarajan, J.Glorium Arulraj, S.Perumal, Physica B 405 (2010) 738-743.

[14]. D. Joseph Daniel, P. Ramasamy, Optical Materials 36 (2014) 971-976.

[15]. N. Manonmani, C.K. Mahadevan, V. Umayorubhagan, Mater. Manuf. Processes 22 (2007) 388.

[16]. K.V. Rao, A. Samakula, J. Appl. Phys. 36 (1965) 2031.

[17]. P.Selvarajan, B.N.Das, H.B.Gon, K.V. Rao, J. Mater. Sci. 29 (1994) 4061.

[18]. M. Sheik-Bahae, A.A. Said, D.J. Hagan, M.J. Soileau, and E.W. Van Stryland, Opt. Eng. 30, 1228-1235 (1990).

[19]. J.A. Hermann and R.G. McDuff, J. Opt. Soc. Am. B10, 2056-2064 (1993). 